\title{
Information-Stressors and Cancer Patients' Quality of Life: Responses to Deviant Information-Stressors Due to Pre-Postoperative Stage Discordance
}

\author{
Seung Soo Lee ${ }^{1,2}$, Ho Young Chung ${ }^{1,2, *}$, and Oh Kyoung Kwon ${ }^{1,3}$ \\ ${ }^{1}$ Department of Surgery, School of Medicine, Kyungpook National University, ${ }^{2}$ Department of Surgery, Kyungpook National University \\ Hospital, ${ }^{3}$ Gastric Cancer Center, Kyungpook National University Chilgok Hospital, Daegu, Korea
}

This study assessed preoperative quality of life (QoL) of gastric cancer patients exposed to inconsistent illness information by pre-post-operative stage discordance. The impact of information-stressors on patients' QoL was investigated to determine information processing as a potential target for QoL management. Early-stage gastric cancer (EsGC) and late-stage gastric cancer (LsGC) groups based on their final stage were categorized by the consistency of preoperative staging information that was being shared. Those with consistent preoperative staging information were rated as EsGC $(n=1,420)$ and LsGC ( $\mathrm{n=153)}$ controls. EsGC and LsGC patients with misdirected information about their LsGC and EsGC were categorized as EsGC/iLsGC (n=32) and LsGC/iEsGC $(\mathrm{n}=55)$, respectively. Preoperative QoL data was obtained using EORTC QLQ-C30 and -STO22. QoL outcomes of EsGC/iLsGC and LsGC/iEsGC were compared with those of the EsGC and LsGC controls. QoL outcomes of the EsGC/iLsGC group matched that of EsGC control, but were significantly better than those of LsGC control on multiple scales including global health status/QoL, physical/role/social-functioning, and ten symptom scales/items. On the other hand, QoL outcomes of LsGC/iEsGC group were significantly better than those of LsGC control on multiple scales (global health status/QoL, physical/role-functioning, and nine symptom scales/items) while they roughly matched with those of EsGC control. Intensified information-stressors did not exacerbate QoL beyond the influence of the patients' medical condition, while de-intensified information-stressor improved QoL. Fear of negatively impacting QoL should not prevent the sharing of stressful illness information. As the de-intensified information-stressor improves QoL, information processing is recommended as a potential target for QoL management in cancer patients.

Key Words: Health Communication; Neoplasm Staging; Quality of Life; Stomach Neoplasms

This is an Open Access article distributed under the terms of the Creative Commons Attribution Non-Commercial License (http://creativecommons.org/licenses/by-nc/4.0) which permits unrestricted non-commercial use, distribution, and reproduction in any medium, provided the original work is properly cited.

\section{Article History:}

Received December 26, 2019

Revised February 6, 2020

Accepted February 13, 2020

\author{
Corresponding Author: \\ Ho Young Chung \\ Department of Surgery, Kyungpook \\ National University Hospital, 130 \\ Dongdeok-ro, Jung-gu, Daegu \\ 41944, Korea \\ Tel: $+82-53-200-5620$ \\ Fax: +82-53-421-0510 \\ E-mail: hychung@knu.ac.kr
}

\section{INTRODUCTION}

Clinicians have to break news to cancer patients about their illnesses and expected consequences of treatment. While such information has little influence on patients' oncologic status, it could impact their subjective wellness, namely their quality of life (QoL). A previous study has shown significant QoL differences between healthy individuals and preoperative gastric cancer patients. ${ }^{1}$ Seeing as early gastric cancer is unlikely to produce significant symptomatic or physical deteriorations, this finding indicates that QoL alterations might be caused by the conveyance of information on early gastric cancer. This strongly indicates the impact of information-stressors on cancer patients' QoL, further suggesting that information processing may be useful as a potential target for QoL management.

Information processing in cancer patients involves inter- 
personal transfer of information and personal interpretation of the transferred information. Ever since the growth of medical science, clinicians have been searching for suitable ways to manage interpersonal transfer of stressful information to cancer patients. This remains a dilemma in everyday practice. ${ }^{2-6}$ Without a sufficient understanding of how stressful information affects patients' QoL, decisions on how it should be communicated have been primarily based on information-sharing rules and policies as well as clinicians' personal and cultural convictions. Since rules or policies and personal/cultural convictions often conflict with each other, some patients are given information emphasizing every possible negative outcome, including those that are highly unlikely to arise, while others might receive information emphasizing the most positive aspects.

Recent development of QoL tools in the form of questionnaires with continuous variable outcomes has been especially beneficial for conducting QoL comparisons between different cohorts. ${ }^{7-9}$ However, studying patients' QoL responses according to the nature of shared illness information has further problems to address. First, we must find a way to categorize such information in terms of the degree of stress it will likely inflict. Second, ethical and trust standards in both clinical and research environments must not be violated by altering the nature of given information.

While it is very unlikely for modern medical science to permit deliberate alteration of given information to ameliorate the recipient patients' stress levels, unintended alteration has been occurring in daily practice when pre-postoperative stage discordances occur. Determination of the preoperative stage is usually based on results of imaging studies such as abdominal computed tomography while that of the postoperative stage is based on surgical observation and pathological findings of surgical specimens. Pre-postoperative stage discordance occurs when intra/ postoperative surgical/pathological findings do not match with preoperative image findings, ${ }^{10-12}$ potentially causing unintentional exposure of different patients with the same cancer stage to different illness-related advice during the preoperative period. While QoL is generally known to deteriorate with cancer progression, ${ }^{13,14}$ assessing preoperative QoL of those with and without incorrect stage information associated with pre-postoperative stage discordance would allow us to verify the impact of physician-provided information on patients' QoL.

The objective of the present study was to investigate how the nature of stressing-information might affect cancer patients' QoL. Thus, this study compared the QoL of gastric cancer patients exposed to inconsistent illness information associated with pre-postoperative stage discordance. A means for how stressful information should be processed to best benefit cancer patients based on actual QoL is also discussed.

\section{MATERIALS AND METHODS}

\section{Study design and participants}

With discord often found between the postoperative pathological stage and the preoperative clinical stage, some cancer patients are exposed to misdirected or deviant illness information during the preoperative period. Thus, early-stage gastric cancer (EsGC) and late-stage gastric cancer (LsGC) groups according to their final stages were categorized by the consistency of preoperative/postoperative staging information that was being shared. Those with consistent preoperative staging information were rated as EsGC and LsGC controls. EsGC patients with misdirected (intensified) preoperative information of LsGC (iLsGC) and LsGC patients with misdirected (de-intensified) information of EsGC (iEsGC) were rated as EsGC/iLsGC and LsGC/iEsGC groups, respectively (Fig. 1A). Therefore, patients were divided into four groups: EsGC control, EsGC/iLsGC, LsGC control, and LsGC/ iEsGC. QoL scales influenced by cancer progression were identified first. Using identified QoL scales, the QoL of each group with misdirected information (EsGC/iLsGC and LsGC/iEsGC) was compared with that of EsGC and LsGC controls (Fig. 1B).

Stage grouping was based on the 7th edition of the Union for International Cancer Control (UICC) classification. Stage IV patients were not considered eligible. This is because they are not usually considered for surgery. Seeking to eliminate the uncertainty of SI shifting through minor discordances between stages I/II or II/III, those with preoperative or postoperative stage II cancer were not included. QoL data was obtained from patients admitted for surgery at our hospitals between 2012 and 2016, yielding an available sample of 1,933 patients.

Patients with comorbidities that could influence their QoL were excluded. A total of 136 patients were excluded. They had the following conditions: cardiac $(n=61)$, cerebrovascular $(n=45)$, renal $(n=11)$, hepatic $(n=7)$, psychological $(n=7)$, neurologic $(n=3)$, and immunologic $(n=2)$ disease. Those with other malignancies $(n=61)$, previous gastric surgeries $(n=24)$, previous endoscopic treatment for gastric cancer $(n=47)$, and neoadjuvant chemotherapy $(n=5)$ were also excluded. After these exclusions, data from 1,660 of 1,933 patients were available for final analyses. Their preoperative QoL and clinical data were retrospectively evaluated after obtaining approval of this study from our institutional review board (IRB No. KNUH 2017-08-007).

\section{QoL assessment}

Preoperative QoL data was obtained from gastric cancer patients upon their admission for surgery. The European Organisation for Research and Treatment of Cancer (EORTC) provides quality of life questionnaires (QLQs) in multiple languages. ${ }^{15-17}$ The Korean version of the EORTC QLQ core module -C30 and the gastric cancer specific module -STO22 were used to assess QoL. ${ }^{18}$ Following the 


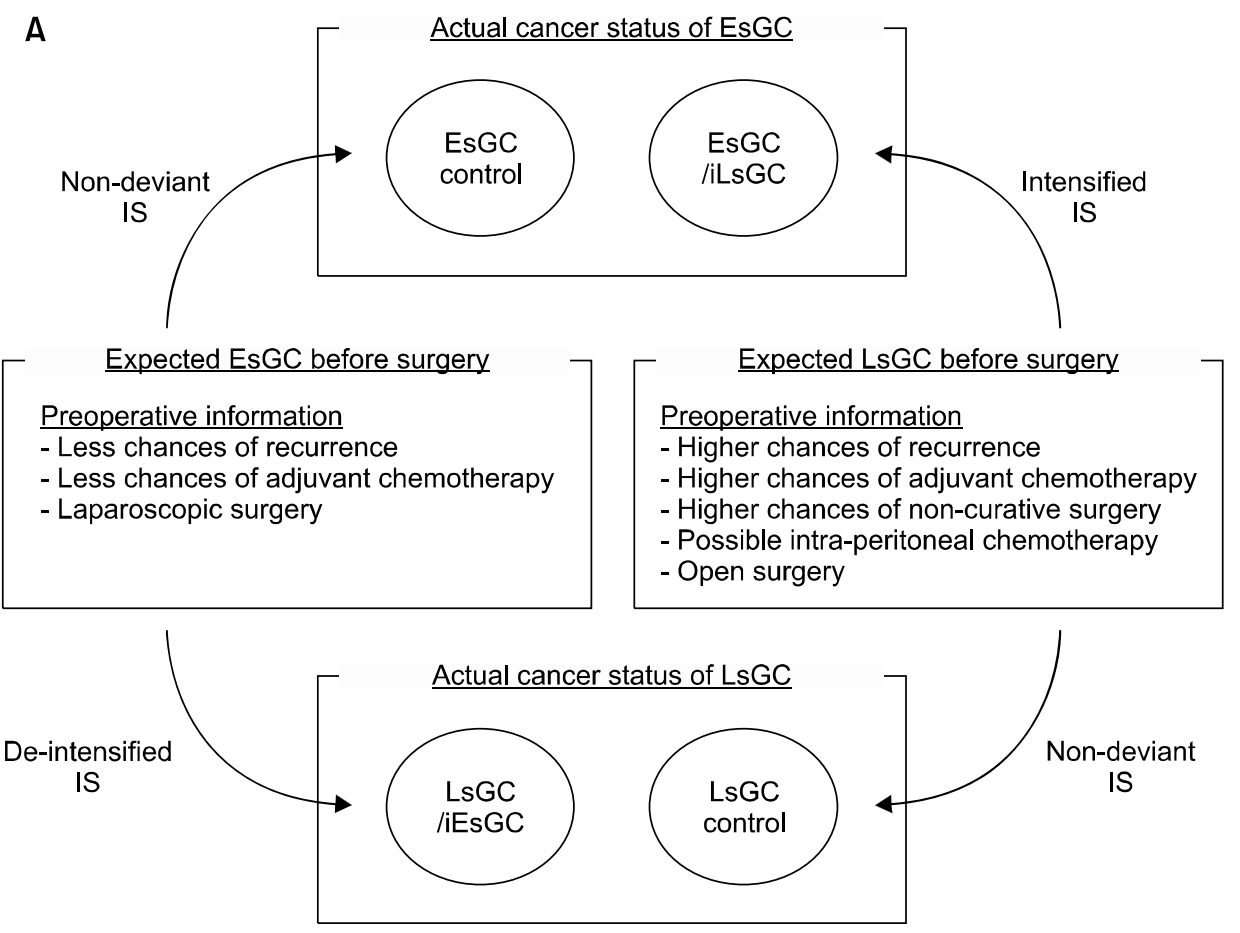

B

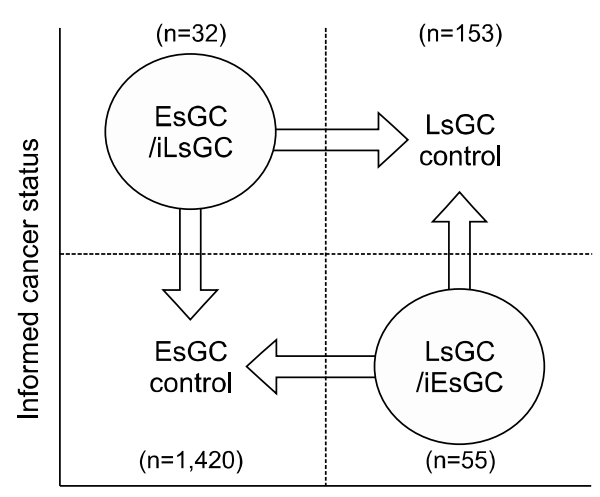

Actual cancer status
FiG. 1. Study design. (A) Overview of consistency rating of preoperative information-stressor, given the stage discordance between pre/post-operative stages. (B) Comparison diagram. IS: information-stressor, EsGC: early-stage gastric cancer, LsGC: late-stage gastric cancer, iEsGC: information of earlystage gastric cancer, iLsGC: information of late-stage gastric cancer.
EORTC's manual, self-administered patient responses to 52 items were transformed into 24 scale scores, ranging from 0 to 100 .

\section{Statistical analysis}

Analyses of variance (ANOVA) and Chi-square tests were used to compare patients' demographics and clinical characteristics. Student's $t$ tests were used to compare QoL scores of EsGC/iLsGC and LsGC/iEsGC with those of EsGC and LsGC controls. A p-value of less than 0.05 was considered statistically significant. In the case of the QoL comparisons of EsGC/iLsGC and LsGC/iEsGC, the false discovery rate (FDR) method for correction of multiple testing was applied to the p-value to account for multiplicity bias. A p-value less than 0.05 after correction by the FDR was considered statistically significant. All statistical analyses were conducted using the Statistical Package for the Social Sciences (SPSS), version 22.0 (IBM SPSS, Armonk, NY, USA).

\section{RESULTS}

\section{Sample characteristics: demographics}

The entire patient group was comprised of 1,082 men and 578 women $(\mathrm{M}: \mathrm{F}=1.87: 1)$. Their mean age was $60.7 \pm 11.9$ years. Among patients with EsGC, there were 1,420 patients for the EsGC control group and 32 patients for the EsGC/iLsGC group. Among those with LsGC, there were 153 patients for the LsGC control group and 55 for the LsGC/iEsGC group. Sample sizes of 44 and 220 were calculated based on independent $t$ test, assuming the QoL difference on at least one of those scales to be 15 points in means and 20 points in standard deviations. Having the total number of tests of 84 and a sample size ratio of 1:5, the test power was 0.80 with a significance level of 0.0006 . The sample size was not quite as large as calculated in EsGC/iLsGC. However, it was expected to maintain a test power to a certain degree. Patient characteristics are summarized in Table 1. Cancer location differed significantly, with the 
TABLE 1. Characteristics of patients

\begin{tabular}{|c|c|c|c|c|c|}
\hline & \multicolumn{2}{|c|}{ EsGC } & \multicolumn{2}{|c|}{ LsGC } & \multirow{2}{*}{ p-value } \\
\hline & EsGC control $^{\mathrm{a}}(\mathrm{n}=1,420)$ & EsGC/iLsGC $^{\mathrm{b}}(\mathrm{n}=32)$ & $\mathrm{LsGC}$ control $^{\mathrm{a}}(\mathrm{n}=153)$ & $\mathrm{LsGC} / \mathrm{iEsGC}^{\mathrm{b}}(\mathrm{n}=55)$ & \\
\hline Age, years & & & & & 0.571 \\
\hline Mean & 60.7 & 58.8 & 61.7 & 60.3 & \\
\hline SD & 11.8 & 12.6 & 11.9 & 12.9 & \\
\hline Gender & & & & & 0.079 \\
\hline Female & $510(35.9)$ & $10(31.3)$ & $39(25.5)$ & $19(34.5)$ & \\
\hline Male & $910(64.1)$ & $22(68.8)$ & $114(74.5)$ & $36(65.5)$ & \\
\hline Location of cancer & & & & & $<0.001$ \\
\hline Upper & $134(9.4)$ & $4(12.5)$ & $42(27.5)$ & $16(29.1)$ & \\
\hline Middle & $319(22.5)$ & $9(28.1)$ & $32(20.9)$ & $13(23.6)$ & \\
\hline Lower & $967(68.1)$ & $19(59.4)$ & $79(51.6)$ & $26(47.3)$ & \\
\hline
\end{tabular}

EsGC: early-stage gastric cancer, LsGC: late-stage gastric cancer, iEsGC: information of early-stage gastric cancer, iLsGC: information of late-stage gastric cancer, SD: standard deviation. Values in parentheses are percentages unless otherwise stated. Bold font denotes a significant difference between groups.

${ }^{a}$ Patients without pre-post-operative stage discordance. ${ }^{b}$ Patients with pre-post-operative stage discordance.

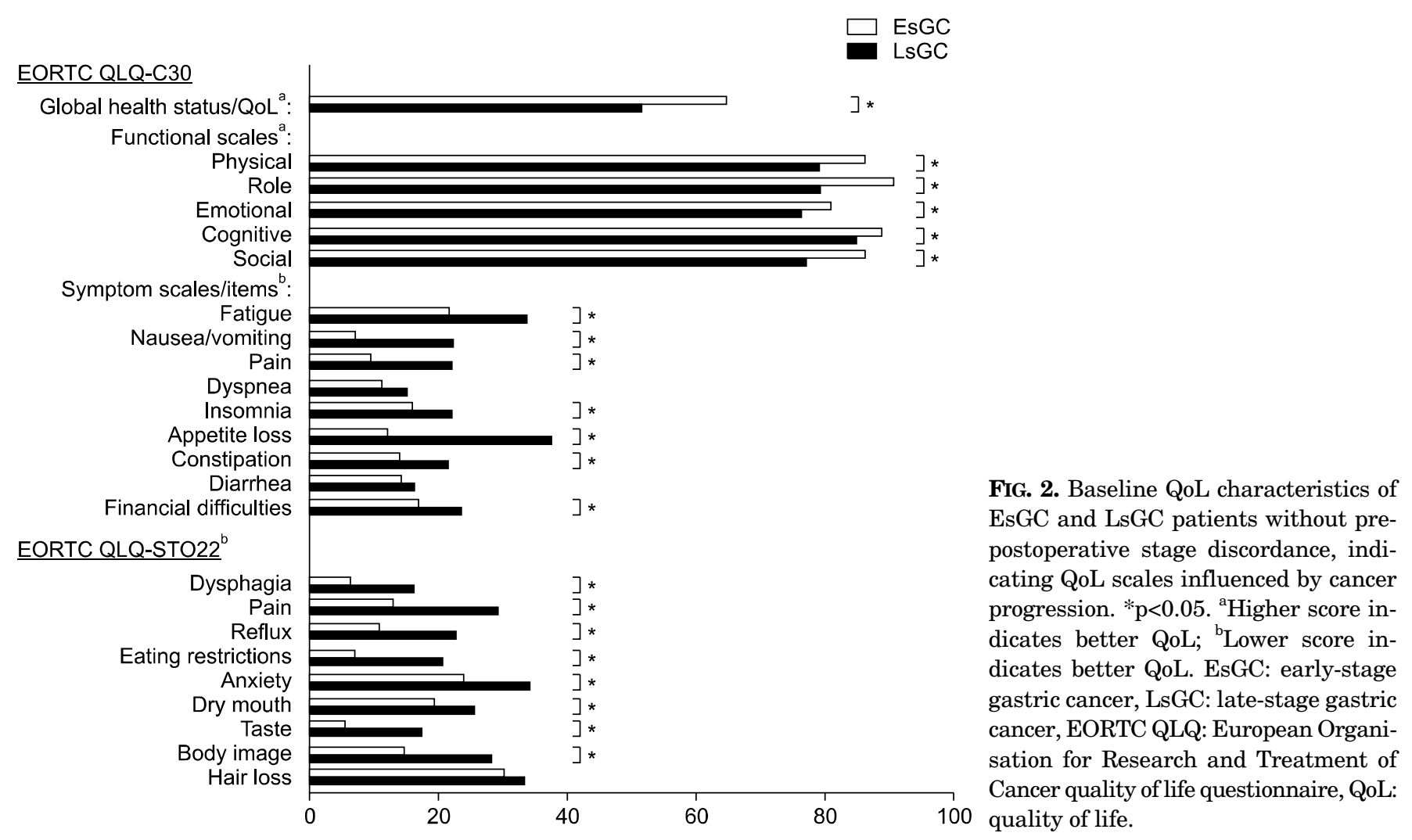

late-stage gastric cancer group showing a higher rate of cancer involving the upper part of the stomach $(\mathrm{p}<0.001)$.

\section{Sample characteristics: QoL by cancer progression}

When QoL trends by cancer progression were analyzed for patients without pre-postoperative stage discordance, the LsGC control group exhibited significantly worse QoL than the EsGC control group on multiple scales of the EORTC QLQ-C30: global health status/QoL, physical functioning, role functioning, emotional functioning, cog- nitive functioning, social functioning, fatigue, nausea/vomiting, pain, insomnia, appetite loss, constipation, and financial difficulties (Fig. 2). Moreover, the LsGC control group exhibited significantly worse QoL than the EsGC control group for eight of nine scales of the EORTC QLQ-STO22: dysphagia, pain, reflux, eating restrictions, anxiety, dry mouth, taste, and body image. 


\section{QoL responses to an intensified information-stressor: EsGC/iLsGC}

Compared to the EsGC control group, there were no significant QoL differences (Table 2).

When comparisons were made with the LsGC control group, the EsGC/iLsGC group tended to have better QoL on multiple scales of the EORTC QLQ-C30 (global health status/QoL, physical functioning, role functioning, social functioning, fatigue, nausea/vomiting, pain, appetite loss, and financial difficulties) and the EORTC QLQ-STO22 (dysphagia, pain, reflux, eating restrictions, anxiety, and taste) despite being exposed to the intensified informationstressor of LsGC. After correction using FDR, those differences, except for anxiety scale of the EORTC QLQ-STO22, remained significant: global health status/QoL (adj. p=0.012), physical functioning (adj. p=0.007), role functioning (adj. $\mathrm{p}=0.006)$, social functioning (adj. $\mathrm{p}=0.003)$, fatigue $(\operatorname{adj} . \mathrm{p}=$ 0.019), nausea/vomiting (adj. p=0.003), pain (adj. p=0.004), appetite loss (adj. p<0.001), financial difficulties (adj. $\mathrm{p}=$ 0.007), dysphagia (adj. p=0.004), pain (adj. p=0.017), reflux (adj. $\mathrm{p}=0.002)$, eating restrictions (adj. $\mathrm{p}<0.001)$, and taste (adj. $\mathrm{p}=0.004)$.

\section{QoL responses to a de-intensified information-stressor: LsGC/iEsGC}

Compared to the EsGC control, the LsGC/iEsGC group showed no significant QoL difference after correction by FDR, although the LsGC/iEsGC group tended to have worse QoL on some scales: pain, dysphagia, and taste (Table 2).

Compared to the LsGC control group, the LsGC/iEsGC group tended to have better QoL on multiple scales of the EORTC QLQ-C30 (global health status/QoL, physical functioning, role functioning, social functioning, fatigue, nausea-vomiting, pain, appetite loss, and constipation) and EORTC QLQ-STO22 (pain, reflux, eating restrictions, anxiety, and body image). After correction by FDR, those differences, except for social functioning and pain of the EORTC QLQ-C30, remained significant: global health status/QoL (adj. p=0.007), physical functioning (adj. p=0.001), role functioning (adj. $\mathrm{p}<0.001$ ), fatigue (adj. $\mathrm{p}=0.002$ ), nausea-vomiting (adj. p=0.002), appetite loss (adj. $\mathrm{p}<0.001)$, constipation (adj. p=0.007), pain (adj. p<0.001), reflux (adj. $\mathrm{p}<0.001$ ), eating restrictions (adj. $\mathrm{p}=0.017$ ), anxiety (adj. $\mathrm{p}=0.019)$, and body image $(\operatorname{adj} . \mathrm{p}=0.004)$.

TABLE 2. QoL of patients with misdirected staging information (EsGC/iLsGC and LsGC/iEsGC) compared to EsGC and LsGC controls by selected scales of the EORTC QLQs

\begin{tabular}{|c|c|c|c|c|c|c|c|c|c|c|c|c|}
\hline & \multicolumn{4}{|c|}{ Control } & \multicolumn{8}{|c|}{ Misdirected information Group } \\
\hline & \multicolumn{2}{|c|}{ EsGC (A) } & \multicolumn{2}{|c|}{ LsGC (B) } & \multicolumn{4}{|c|}{ EsGC/iLsGC } & \multicolumn{4}{|c|}{ LsGC/iEsGC } \\
\hline & $\mathrm{N}$ & Mean (SD) & $\mathrm{N}$ & Mean (SD) & $\mathrm{N}$ & Mean (SD) & $\mathrm{p}(\mathrm{vs} \mathrm{A})$ & $\mathrm{p}(\mathrm{vs} B)$ & $\mathrm{N}$ & Mean (SD) & $\mathrm{p}(\mathrm{vs} A)$ & $p(\operatorname{vs~B})$ \\
\hline Global health status/QoL ${ }^{a}$ & 1350 & $64.3(22.1)$ & 143 & $51.3(27.1)$ & 32 & $66.7(21.9)$ & 0.556 & 0.003* & 52 & $65.1(23.5)$ & 0.818 & $0.001 *$ \\
\hline Physical functioning $^{\mathrm{a}}$ & 1404 & $85.6(15.1)$ & 150 & $78.6(20.3)$ & 32 & $87.9(13.1)$ & 0.389 & $0.002 *$ & 55 & $88.2(13.2)$ & 0.201 & $<0.001 *$ \\
\hline Role functioning $^{\mathrm{a}}$ & 1403 & $90.1(17.5)$ & 149 & $78.7(27.4)$ & 32 & $91.7(17.5)$ & 0.613 & 0.001* & 55 & $92.7(15.6)$ & 0.270 & $<0.001^{*}$ \\
\hline Emotional functioning $^{a}$ & 1403 & $80.4(19.3)$ & 150 & $75.9(21.2)$ & 32 & $82.6(17.0)$ & 0.527 & 0.098 & 55 & $80.9(20.8)$ & 0.840 & 0.133 \\
\hline Cognitive functioning $^{\mathrm{a}}$ & 1404 & $88.1(15.4)$ & 150 & $84.2(20.0)$ & 32 & $90.1(17.9)$ & 0.473 & 0.127 & 55 & $88.5(16.3)$ & 0.863 & 0.159 \\
\hline Social functioning ${ }^{\mathrm{a}}$ & 1395 & $85.6(20.7)$ & 150 & $76.7(28.1)$ & 32 & $89.6(15.1)$ & 0.275 & $<0.001^{*}$ & 55 & $83.9(21.0)$ & 0.567 & 0.048 \\
\hline Fatigue $^{\mathrm{b}}$ & 1401 & $21.8(18.9)$ & 149 & $33.6(24.9)$ & 32 & $20.1(23.3)$ & 0.621 & 0.006* & 55 & $19.3(18.7)$ & 0.331 & $<0.001 *$ \\
\hline Nausea and vomiting ${ }^{\mathrm{b}}$ & 1403 & $7.3(14.1)$ & 151 & $22.4(27.8)$ & 32 & $8.9(16.4)$ & 0.547 & $<0.001^{*}$ & 55 & $9.4(19.4)$ & 0.439 & $<0.001^{*}$ \\
\hline Pain $^{\text {b }}$ (QLQ-C30) & 1403 & $9.8(16.2)$ & 150 & $22.2(23.3)$ & 32 & $11.5(13.0)$ & 0.568 & 0.001* & 55 & $14.5(19.3)$ & 0.035 & $\mathbf{0 . 0 3 0}$ \\
\hline Insomnia $^{\mathrm{b}}$ & 1396 & $16.1(24.2)$ & 149 & $22.1(29.2)$ & 32 & $13.5(25.2)$ & 0.548 & 0.123 & 55 & $16.4(24.7)$ & 0.947 & 0.193 \\
\hline Appetite loss ${ }^{\mathrm{b}}$ & 1396 & $12.4(21.5)$ & 150 & $37.6(37.1)$ & 32 & $12.5(18.5)$ & 0.973 & $<0.001^{*}$ & 55 & $15.2(24.7)$ & 0.348 & $<0.001^{*}$ \\
\hline Constipation $^{\mathrm{b}}$ & 1397 & $14.1(23.7)$ & 150 & $21.6(27.9)$ & 31 & $17.2(22.6)$ & 0.466 & 0.416 & 53 & $10.7(18.2)$ & 0.195 & $0.002 *$ \\
\hline Financial difficulties $^{\mathrm{b}}$ & 1369 & $17.1(26.2)$ & 144 & $23.6(30.2)$ & 32 & $10.4(17.8)$ & 0.046 & $0.002 *$ & 53 & $20.8(26.3)$ & 0.322 & 0.544 \\
\hline Dysphagia $^{\mathrm{b}}$ & 1403 & $6.6(10.8)$ & 151 & $16.3(22.3)$ & 32 & $7.6(9.5)$ & 0.579 & $0.001^{*}$ & 55 & $13.5(20.9)$ & 0.017 & 0.418 \\
\hline Pain $^{b}$ (QLQ-STO22) & 1403 & $13.1(15.0)$ & 151 & $29.3(22.9)$ & 32 & $16.9(17.5)$ & 0.160 & $0.005^{*}$ & 55 & $15.6(15.7)$ & 0.242 & $<0.001 *$ \\
\hline $\operatorname{Reflux}^{\mathrm{b}}$ & 1405 & $11.2(15.4)$ & 149 & $22.8(24.7)$ & 32 & $10.4(13.5)$ & 0.786 & $<0.001 *$ & 55 & $10.5(14.7)$ & 0.756 & $<0.001 *$ \\
\hline Eating restrictions ${ }^{\mathrm{b}}$ & 1404 & $7.4(11.4)$ & 150 & $20.8(22.5)$ & 32 & $8.1(11.7)$ & 0.727 & $<0.001^{*}$ & 55 & $12.1(18.1)$ & 0.061 & $0.005^{*}$ \\
\hline Anxiety $^{b}$ & 1402 & $24.0(20.5)$ & 150 & $34.1(24.0)$ & 32 & $25.0(21.5)$ & 0.787 & 0.048 & 55 & $24.0(20.0)$ & 0.991 & $0.006^{*}$ \\
\hline Dry mouth ${ }^{\mathrm{b}}$ & 1397 & $19.6(24.6)$ & 149 & $25.7(29.0)$ & 32 & $16.7(25.4)$ & 0.499 & 0.104 & 55 & $21.8(53.0)$ & 0.762 & 0.504 \\
\hline Taste $^{b}$ & 1393 & $5.8(15.0)$ & 148 & $17.6(27.1)$ & 32 & $6.3(13.2)$ & 0.857 & $0.001 *$ & 54 & $12.3(22.7)$ & 0.039 & 0.208 \\
\hline Body image $^{b}$ & 1395 & $14.8(23.2)$ & 149 & $28.2(29.7)$ & 32 & $17.7(25.4)$ & 0.489 & 0.065 & 55 & $14.5(22.9)$ & 0.927 & $0.001 *$ \\
\hline
\end{tabular}

QoL: quality of life, EsGC: early-stage gastric cancer, LsGC: late-stage gastric cancer, iEsGC: information of early-stage gastric cancer, iLsGC: information of late-stage gastric cancer, EORTC QLQ: European Organisation for Research and Treatment of Cancer quality of life questionnaire. Bold font denotes $\mathrm{p}<0.05$. *Significant difference with $\mathrm{p}<0.05$ after correction by false discovery rate method to account for the total number of tests of $84 .{ }^{a}$ Higher score representsbetter QoL; ${ }^{b}$ Lower score represents better QoL. 


\section{DISCUSSION}

We investigated the impact of information-stressors on cancer patients' QoL by comparing groups of cancer patients preoperatively exposed to inconsistent illness information due to pre-postoperative stage discordance. Both the EsGC/iLsGC and LsGC/iEsGC groups exhibited QoL outcomes that were similar to EsGC control and better that those of LsGC control. When somatic input and informational input yielded different levels of stresses, cancer patients exhibited a compliance with the input which was less stressful. Our study suggests a reasonable basis, at least in the study region, for physicians to be less concerned over QoL deterioration from sharing worrisome information regarding consequences of gastric cancer and its treatment. Although medicine has become globalized over the past century, regional and cultural influences remain. While full disclosure of illnesses and related outcomes are much valued in some countries, the culture and value systems of other countries favor non-disclosure of intensely stressful information to patients to avoid damaging their mental and spiritual well-being. ${ }^{2,4,19,20}$ When breaking bad news, physicians must consider both global trends and regional culture, resulting in a dilemma for those physicians from societies where patients' emotional stability or comfort is much valued. In Korea, patients' emotional stability, especially emotional stability of elderly patients, is an important part of the value system. Consequently, sometimes physicians have to make difficult and unwanted decisions on what information to share directly with the patient and indirectly with family members. However, in our study, an intense information-stressor did not result in QoL deterioration beyond the impact of patients' medical condition. This suggests that intense illness information can be shared with patients without significantly harming their subjective well-being.

Unlike intensified information-stressors without significant detrimental impact on patient's QoL, de-intensified information-stressors were associated with significant QoL improvement. This has significant implications for modern medical science in which patient QoL is an important part of cancer survivorship care. Theoretically, QoL improvement could be expected from directly altering illness information to understate its true seriousness or gravity. However, such direct tampering with information cannot be justified under any circumstances. Nevertheless, our results suggest the potential for further conditioning of patients' QoL by focusing on the processing of information-stressors that have already been shared.

Our study has some limitations. As QoL is meant to represent one's perceived position in life in the context of culture and value systems, ${ }^{21}$ analysis of QoL patterns requires an understanding of regional characteristics. There are two regional characteristics of our study location that should be addressed: 1) a high prevalence of gastric cancer; and 2) a well-established health screening system with regular endoscopic examinations that enables early detection of gastric cancer. ${ }^{22}$ Due to these characteristics, early gastric cancer survivors are among the most frequently encountered cancer survivors in this region. This could lead the regional population to perceive gastric cancer as being readily conquerable with favorable outcomes. Although this study's patients exhibit no QoL deterioration from intensification of information-stressor, this finding may not be generalizable to patients in regions with other epidemiological traits and medical systems, especially where gastric cancer is less prevalent and endoscopic screening is less common. Future studies encompassing regions with different characteristics are recommended.

Second, patients with perioperative stage II or stage IV gastric cancer were not included in this study. While stage IV gastric cancer patients were excluded because surgery was precluded in such cases by our practice guidelines, the exclusion of those with stage II cancer would require justification. Although it may seem more reasonable to study the QoL of stage II patients with deviant information to either stage I or stage III cancer, illness information may differ due to stage discordances between two succeeding stages. To identify groups with significant differences in shared information, stage I and stage III groups with deviant information were chosen as subjects of this study.

Third, theoretically, it should be more convincing to determine QoL re-distribution after subjects are exposed to corrected information on cancer status. However, in an actual practice environment, QoL re-distribution could only be studied after the final confirmation of cancer status following surgery. One may argue that postoperative QoL represents altered QoL with reduced cancer burden from surgical resection and that it has low test power for QoL difference in relation to cancer progression. Nevertheless, in our study, postoperative QoL was unavailable from a significant portion of our study groups as they were being referred to medical oncologists for adjuvant chemotherapy.

Finally, the sample size in the EsGC/iLsGC groups was not as large as expected. There might be some unrecognized QoL differences during comparisons including EsGC/iLsGC. To identify specific QoL scales influenced by intensified information stressor, a further study with a larger number of EsGC/iLsGC patients is needed.

Our study highlights the potential for QoL management in cancer patients by targeting information processing beyond symptomatic and functional measures. After identifying the potential for QoL management by controlling the information-stressor itself, there is a strong possibility for its personal interpretation to become the next potential target. Psychiatric counseling has been suggested as a means to improve patient QoL in previous studies. ${ }^{7}$ However, those suggestions were based on terminological impression of QoL scales with psychiatric relevance. This study's principal contributions are diversifying potential targets for QoL management in cancer patients and providing supporting data for future QoL studies involving information processing (e.g., psychiatric counseling).

In conclusion, an intensified information-stressor does 
not exacerbate QoL beyond the current presentation while a de-intensified information-stressor ameliorates QoL in cancer patients. This highlights our human nature of preferring a more favorable stressor among implicated stressors. Therefore, reluctance to share stressful information due to worries of overly diminished QoL is unfounded. Processing of already-shared information should be a potential target to achieve further QoL amelioration for cancer patients.

\section{CONFLICT OF INTEREST STATEMENT}

None declared.

\section{REFERENCES}

1. Lee SS, Chung HY, Kwon O, Yu W. Long-term shifting patterns in quality of life after distal subtotal gastrectomy: preoperativeand healthy-based interpretations. Ann Surg 2015;261:1131-7.

2. Konstantis A, Exiara T. Breaking bad news in cancer patients. Indian J Palliat Care 2015;21:35-8.

3. Chittem M, Butow P. Responding to family requests for nondisclosure: the impact of oncologists' cultural background. J Cancer Res Ther 2015;11:174-80.

4. Seifart C, Hofmann M, Bär T, Riera Knorrenschild J, Seifart U, Rief W. Breaking bad news-what patients want and what they get: evaluating the SPIKES protocol in Germany. Ann Oncol 2014; 25:707-11.

5. Seyedrasooly A, Rahmani A, Zamanzadeh V, Aliashrafi Z, Nikanfar AR, Jasemi M. Association between perception of prognosis and spiritual well-being among cancer patients. J Caring Sci 2014;3: 47-55.

6. Baile WF, Buckman R, Lenzi R, Glober G, Beale EA, Kudelka AP. SPIKES-A six-step protocol for delivering bad news: application to the patient with cancer. Oncologist 2000;5:302-11.

7. Lee SS, Chung HY, Kwon OK, Yu W. Long-term quality of life after distal subtotal and total gastrectomy: symptom- and behavior-oriented consequences. Ann Surg 2016;263:738-44.

8. Kim YW, Baik YH, Yun YH, Nam BH, Kim DH, Choi IJ, et al. Improved quality of life outcomes after laparoscopy-assisted distal gastrectomy for early gastric cancer: results of a prospective randomized clinical trial. Ann Surg 2008;248:721-7.

9. Díaz De Liaño A, Oteiza Martínez F, Ciga MA, Aizcorbe M, Cobo F, Trujillo R. Impact of surgical procedure for gastric cancer on quality of life. Br J Surg 2003;90:91-4.

10. Biron VL, O'Connell DA, Seikaly H. The impact of clinical versus pathological staging in oral cavity carcinoma - a multi-institutional analysis of survival. J Otolaryngol Head Neck Surg 2013; 42:28.

11. Bentrem D, Gerdes H, Tang L, Brennan M, Coit D. Clinical correlation of endoscopic ultrasonography with pathologic stage and outcome in patients undergoing curative resection for gastric cancer. Ann Surg Oncol 2007;14:1853-9.

12. Chen CY, Hsu JS, Wu DC, Kang WY, Hsieh JS, Jaw TS, et al. Gastric cancer: preoperative local staging with 3D multi-detector row $\mathrm{CT}$ - correlation with surgical and histopathologic results. Radiology 2007;242:472-82.

13. Xie Y, Zhao FH, Lu SH, Huang H, Pan XF, Yang CX, et al. Assessment of quality of life for the patients with cervical cancer at different clinical stages. Chin J Cancer 2013;32:275-82.

14. Matsushita T, Matsushima E, Maruyama M. Assessment of peri-operative quality of life in patients undergoing surgery for gastrointestinal cancer. Support Care Cancer 2004;12:319-25.

15. Huijer HA, Sagherian K, Tamim H. Validation of the Arabic version of the EORTC quality of life questionnaire among cancer patients in Lebanon. Qual Life Res 2013;22:1473-81.

16. Zhao H, Kanda K. Translation and validation of the standard Chinese version of the EORTC QLQ-C30. Qual Life Res 2000;9: 129-37.

17. Kobayashi K, Takeda F, Teramukai S, Gotoh I, Sakai H, Yoneda $\mathrm{S}$, et al. A cross-validation of the European Organization for Research and Treatment of Cancer QLQ-C30 (EORTC QLQ-C30) for Japanese with lung cancer. Eur J Cancer 1998;34:810-5.

18. Yun YH, Park YS, Lee ES, Bang SM, Heo DS, Park SY, et al. Validation of the Korean version of the EORTC QLQ-C30. Qual Life Res 2004;13:863-8.

19. Beyraghi N, Mottaghipour Y, Mehraban A, Eslamian E, Esfahani F. Disclosure of cancer information in Iran: a perspective of patients, family members, and health professionals. Iran J Cancer Prev 2011;4:130-4.

20. Montazeri A, Vahdani M, Haji-Mahmoodi M, Jarvandi S, Ebrahimi M. Cancer patient education in Iran: a descriptive study. Support Care Cancer 2002;10:169-73.

21. WHOQOL Group. Study protocol for the World Health Organization project to develop a Quality of Life assessment instrument (WHOQOL). Qual Life Res 1993;2:153-9.

22. Lee HJ, Yang HK, Ahn YO. Gastric cancer in Korea. Gastric Cancer 2002;5:177-82. 